\title{
Large deflection analysis of skew plates under uniformly distributed load for mixed boundary conditions
}

\author{
Debabrata Das ${ }^{*}$, Prasanta Sahoo, Kashinath Saha
}

Department of Mechanical Engineering, Jadavpur University, Kolkata - 700032, INDIA.

"Corresponding Author: e-mail:debudas.ju@gmail.com

\begin{abstract}
The present paper deals with large deflection static behaviour of thin isotropic skew plates under uniformly distributed load for various mixed flexural boundary conditions. A variational method based on the principle of minimization of total potential energy has been used through assumed displacement fields. The results are generated for different possible combinations of clamped and simply supported boundary conditions and presented in normalized load-deflection plane. Individual effects of skew angle, aspect ratio and boundary condition on normalized load-deflection behaviour are separately shown.
\end{abstract}

Keywords: Skew plate; Variational method; Geometric non-linearity; Large deflection

\section{Introduction}

Skew plates are extensively used in various mechanical, civil and aero structures and they are mostly subjected to uniform pressure loading in its transverse faces. Specific application of isotropic skew plates includes aircraft wings and aircraft tail-fins. The edges of these plates are often so mounted that their boundaries can be assumed to be equivalent to various classical flexural boundary conditions. As static behaviour of the skew plates has significant contribution for its mechanical design, the present paper deals with the simulation of static behaviour of skew plates under uniform pressure loading.

Research work on predicting static behaviour and stability of skew plates had been carried out by various researchers over the past three decades. The published research works in this field encompass a wide variety of practical problems. The static behaviour of orthotropic skew plates had been investigated by Alwar and Rao $(1973,1974)$ using dynamic relaxation technique and Malekzadeh and Fiouz (2007) using differential quadrature (DQ) approach. The static analysis of isotropic thick skew plates had been carried out by various researchers (Liew and Han, 1997; Muhammad and Singh, 2004). The buckling analysis of thick skew plates had been carried out by Xiang et al. (1995) and Liew et al. (2004). Recently, Prakash et al. (2008) analyzed thermal postbuckling behaviour of skew plates having functionally graded materials while Daripa and Singha (2009) studied the influence of corner stresses on the stability behaviour of composite skew plates.

Chia (1980) investigated large deflection elastic behaviour of homogenous isotropic and laminated anisotropic skew plates by an analytical method. Rajamohan and Raamachandran (1997) used Boundary Element Method to obtain a fundamental solution for the analysis of isotropic skew plates under transverse loading. Duan and Mahendran (2003) had developed a new non-linear quadrilateral hybrid/mixed shell element using oblique coordinate systems to investigate the large deflection behavior of skew plates under uniformly distributed and concentrated loads. Singh and Elaghabash (2003) developed a numerical method for geometrically nonlinear static analysis of thin plates having quadrangular boundary with four straight edges. Recently, Das et al. (2009) investigated the static behaviour of clamped and simply supported skew plates under pressure loading through a numerical domain mapping technique.

The present paper is an extension of the earlier work by Das et al. (2009) to analyse the large deflection static behaviour of isotropic skew plates under uniform pressure loading. As compared to the earlier work which deals with the skew plates of clamped and simply supported boundaries, the present work deals with the same with all possible combinations of clamped and simply supported boundaries. The formulation is based on the total potential energy of the system and the governing equations are 
obtained using variational principle. The solution is approximate in nature and is obtained by assuming the displacement fields as finite linear combinations of unknown coefficients and admissible coordinate functions. The entire computation is carried out in a normalized square domain which is obtained using appropriate domain mapping technique. Results are presented in normalized load-deflection plane to show the effect of skew angle, aspect ratio and boundary condition.

\section{Mathematical Formulation}

A skew plate $(a \times b \times t)$ with skew angle $\theta$ is shown in Figure 1(a). It is assumed that the material of the plate is isotropic and homogeneous and retains linear elastic behaviour under large displacement regime. The effect of shear deformation is neglected by assuming the thickness of the plate to be sufficiently small $(t / a \leq 0.02)$.

The physical domain of interest is mapped to the square computational domain in $\xi-\eta$ coordinate system as shown in Figure 2. In this domain, the four straight edges $\xi=0,1$ and $\eta=0,1$ represents the oblique plate geometry and the intersections of constant $\xi$ and constant $\eta$ lines passing through the gauss points are selected as the reference points for computation. The coordinates of all the gauss points in the computational domain are transformed to the $x-y$ plane by suitable grid generation techniques. The relationships used for grid generation are given below:

$$
\begin{aligned}
& x=a \xi+b \eta \sin \theta \\
& y=b \eta \cos \theta
\end{aligned}
$$

The system of governing equations is obtained by applying the variational principle of minimization of total potential energy,

$$
\delta(U+V)=0
$$

where, $U=U_{b}+U_{m}$ is total strain energy in which $U_{b}$ is strain energy due to bending and $U_{m}$ is strain energy due to stretching of its mid-plane, $V$ is the potential energy of the external forces. In case of small deformation, the deflected shape of the midplane can be modeled by the transverse displacement $w$ only. However, for large deformation, in-plane displacement fields $u$ and $v$ are also required for proper representation of the deflected plate geometry, thus producing nonlinearity in straindisplacement relations. It is to be noted here that geometric nonlinearity, i.e., the nonlinear strain-displacement relationship is taken into consideration through in-plane stretching. The expressions of $U_{b}$ and $U_{m}$ are not given here to maintain brevity and can be obtained in Das et al. (2009).

For a plate loaded with uniform transverse pressure $p$ (loading is shown in Figures 1(a)-1(d)), potential energy $V$ is given by,

$$
V=-\int_{0}^{b \cos \theta} \int_{y \tan \theta}^{y \tan \theta+a}(p w) d x d y
$$

The present analysis is carried out for skew plates having mixed clamped (C) and simply supported (S) flexural boundary conditions, namely, CCCS, CSCS, CCSS and CSSS. It must be noted that the abbreviations for denoting the mixed boundary conditions start from the skew side of the plate passing through the origin and proceed in counter clockwise direction as denoted by edge 1, 2, 3 and 4 on the plate boundaries in Figures 1(a)-1(d). As regard to membrane effect, the edges of the plate are modeled as immovable by imposing zero in-plane displacement boundary conditions.

\subsection{Derivation of governing equations:}

The displacement fields $u, v$ and $w$ are expressed by linear combinations of unknown parameters $d_{i}$ and admissible coordinate functions as follows:

$$
\begin{aligned}
& w(\xi, \eta)=\sum_{i=1}^{n w} d_{i} \phi_{i}(\xi, \eta), \\
& u(\xi, \eta)=\sum_{i=n w+1}^{n w+n u} d_{i} \alpha_{i-n w}(\xi, \eta), \\
& v(\xi, \eta)=\sum_{i=n w+n u+1}^{n w+n u+n v} d_{i} \beta_{i-n w-n u}(\xi, \eta)
\end{aligned}
$$

where, $\phi(\xi, \eta), \alpha(\xi, \eta)$ and $\beta(\xi, \eta)$ are sets of orthogonal coordinate functions and $n w, n u$ and $n v$ are number of functions used for approximating $u, v$ and $w$ respectively. 


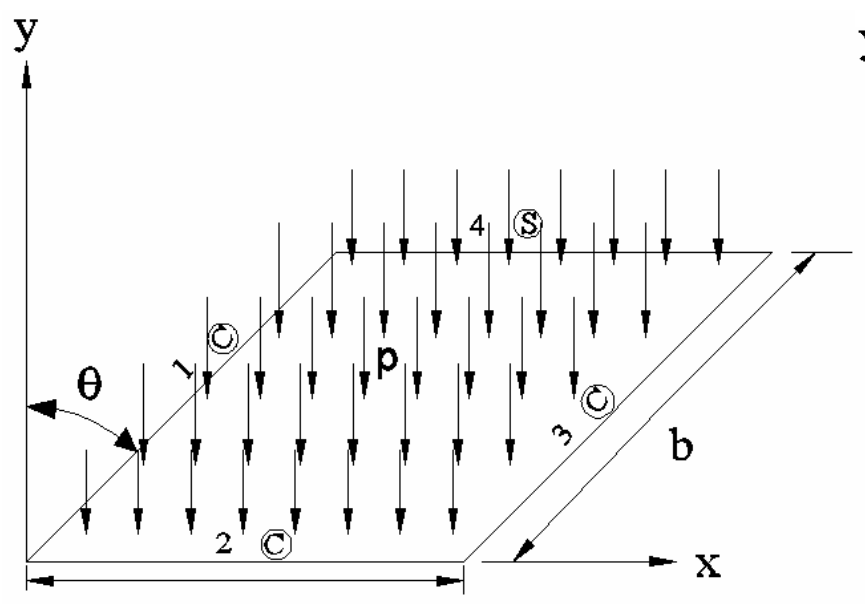

a

(a) CCCS

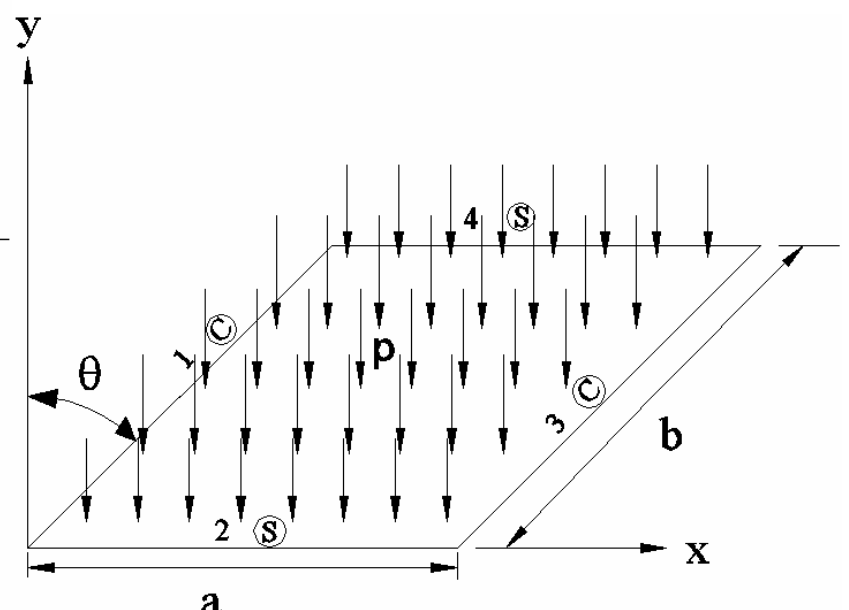

(b) CSCS

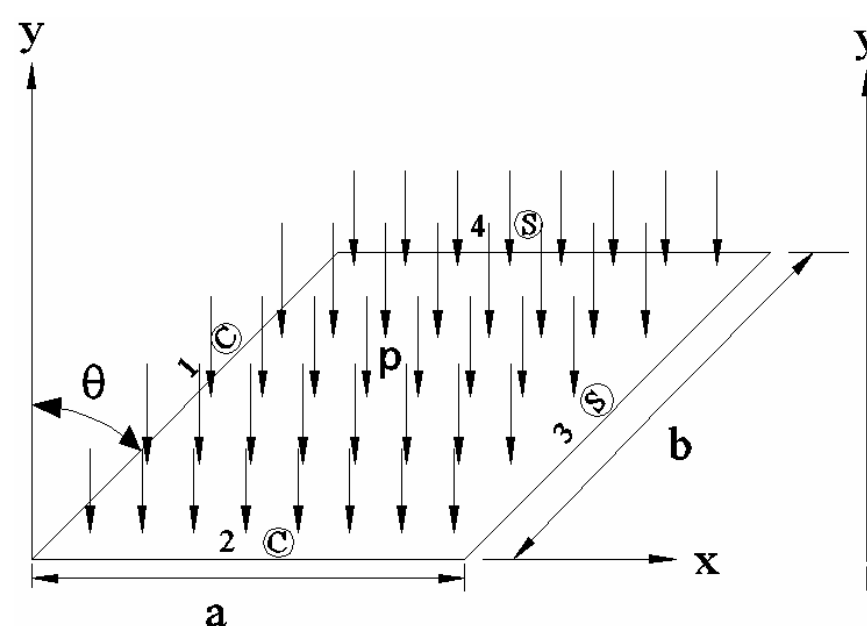

(c) CCSS

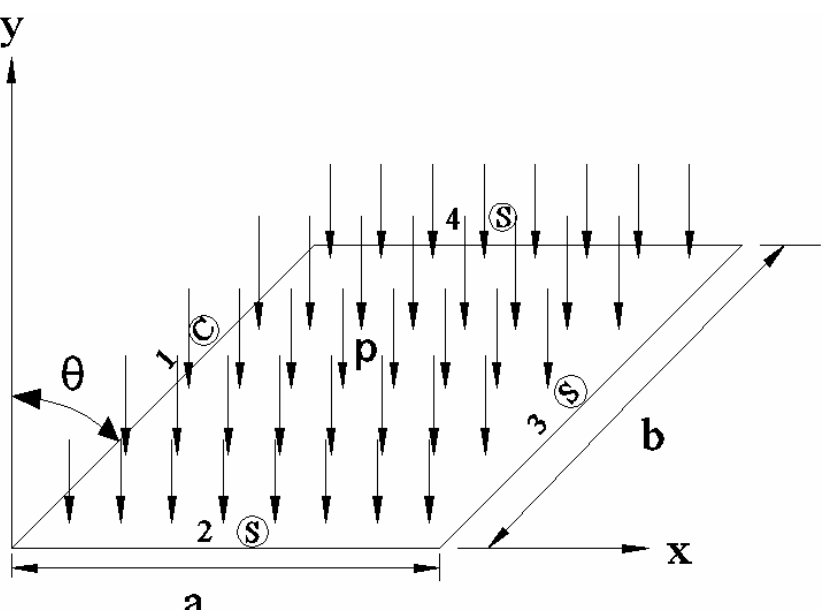

(d) CSSS

Figure 1. Skew plate with uniform pressure loading and various boundary conditions: (a) CCCS, (b) CSCS, (c) CCSS and (d) CSSS.

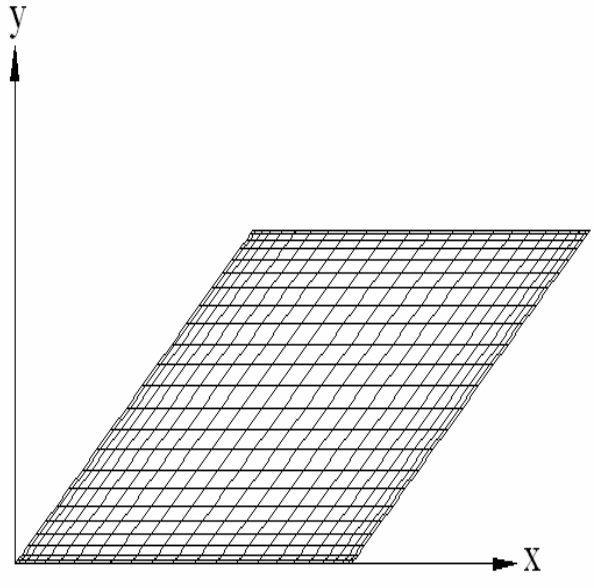

(a) Physical domain

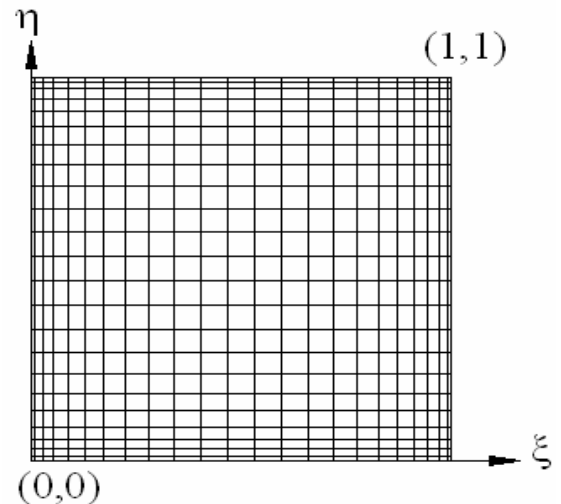

(b) Computational domain

Figure 2. Gauss point locations for skew plate (Das et al., 2009): (a) Physical domain and (b) Computational domain. 
The start functions of these orthogonal sets are selected to satisfy the flexural and membrane boundary conditions of the plate. To cater to the need of numerical scheme, all the start functions are defined in computational domain. The basis functions for the definition of plate deflection $w$ comes from different possible combinations of clamped and simply supported boundary conditions. The functions used as basis or start function for different boundary conditions are shown in Table 1 . The variations of one-dimensional coordinate functions along normalized coordinate are shown in Figure 3 for three different boundary conditions, namely CC, CS and SS. The first three functions (functions 1-3) in terms of increasing order are shown in Figure 3(a), whereas, functions 4-6 are shown in Figure 3(b). The start functions for $u$ and $v$ are selected to satisfy the zero displacement conditions at the boundary and are given in Table 2. The higher order functions are generated following a two dimensional implementation of Gram-Schmidt orthogonalization scheme. Mathematically, clamped and simply supported boundary conditions (with rigid inplane restraint) are given by, $w=0, u=0, v=0$ and $\frac{\partial w}{\partial \xi}=0$ or, $\frac{\partial w}{\partial \eta}=0$ along the plate edges for clamped boundary condition, and
$w=0, u=0, v=0$ and $\frac{\partial^{2} w}{\partial \xi^{2}}=0$ or, $\frac{\partial^{2} w}{\partial \eta^{2}}=0$ along the plate edges for simply supported boundary condition.

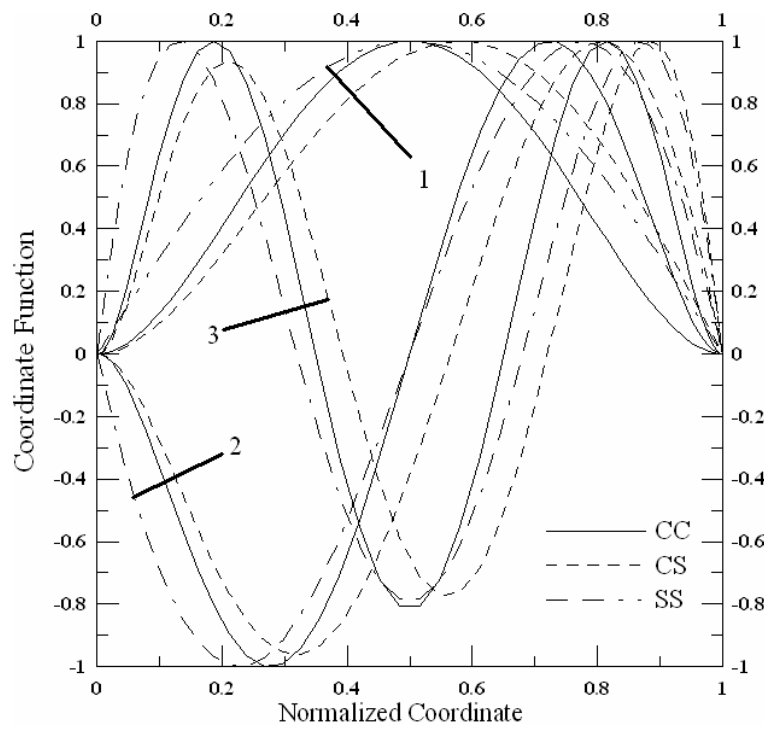

(a) Functions 1-3

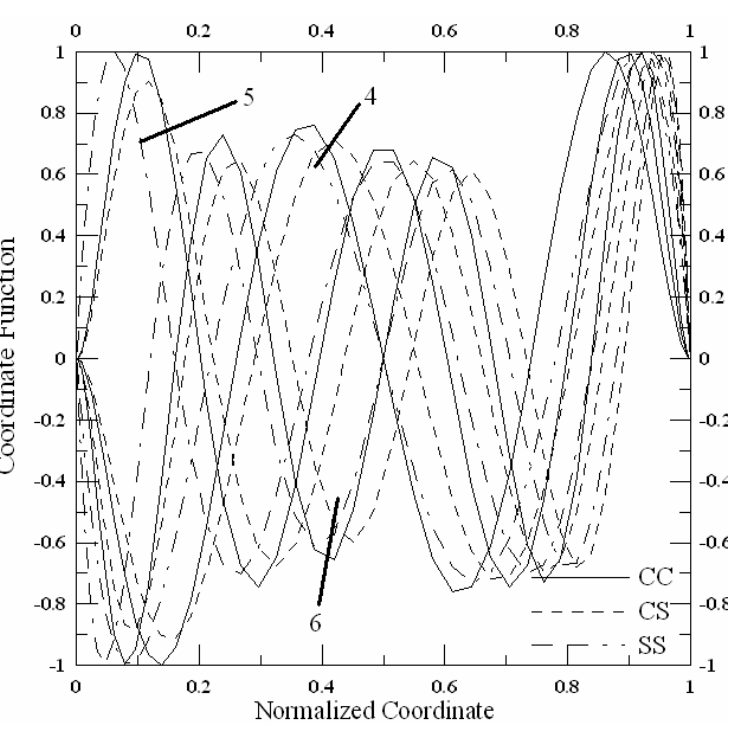

(b) Functions 4-6

Figure 3. Variation of coordinate functions along normalized coordinate: (a) Functions 1-3 and (b) Functions 4-6.

Table 1. Start functions for displacement $w$

\begin{tabular}{|c|c|}
\hline Boundary condition & $\phi_{1}(\xi, \eta)$ \\
\hline CCCS & $\{\xi(1-\xi)\}^{2}\left\{\eta^{2}\left(3-5 \eta+2 \eta^{2}\right)\right\}$ \\
\hline CSCS & $\{\xi(1-\xi)\}^{2}\{\sin (\pi \eta)\}$ \\
\hline CCSS & $\left\{\xi^{2}\left(3-5 \xi+2 \xi^{2}\right)\right\}\left\{\eta^{2}\left(3-5 \eta+2 \eta^{2}\right)\right\}$ \\
\hline CSSS & $\left\{\xi^{2}\left(3-5 \xi+2 \xi^{2}\right)\right\}\{\sin (\pi \eta)\}$ \\
\hline
\end{tabular}

Table 2. Start functions for displacements $u$ and $v$

\begin{tabular}{|c|c|}
\hline Displacement & Start function \\
\hline$u$ & $\alpha_{1}=\{\xi(1-\xi)\}\{\eta(1-\xi)\}$ \\
\hline$v$ & $\beta_{1}=\{\xi(1-\xi)\}\{\eta(1-\xi)\}$ \\
\hline
\end{tabular}




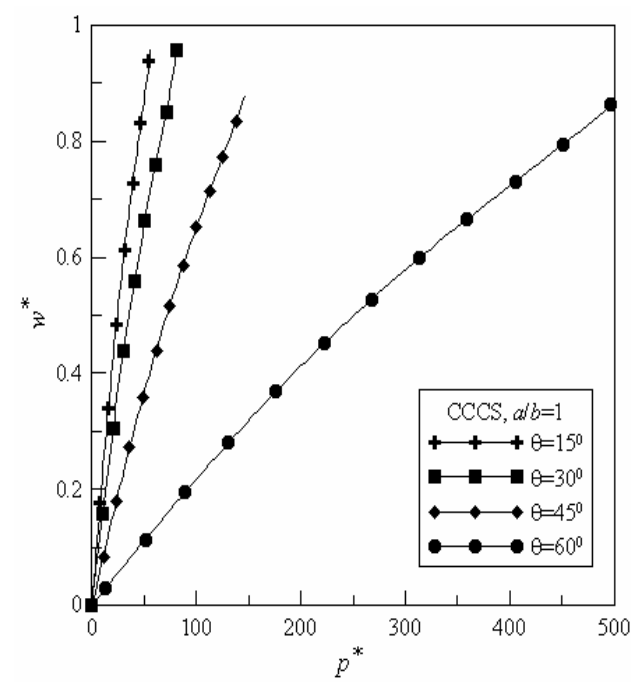

(a) $a / b=1$

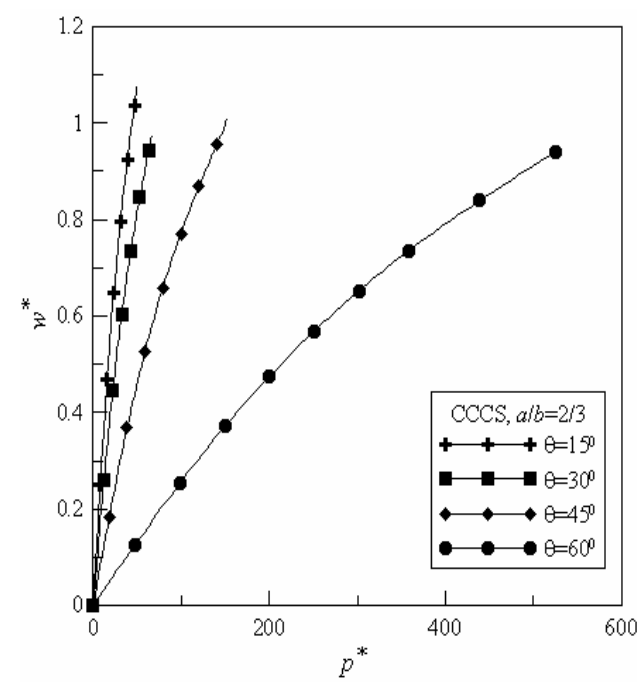

(b) $a / b=2 / 3$

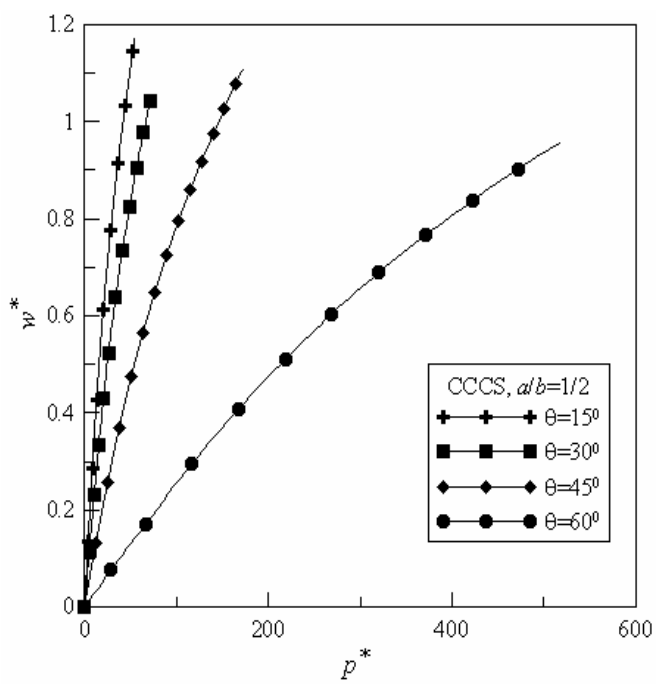

(c) $a / b=1 / 2$

Figure 4. Normalized load-deflection plots of CCCS plate of different skew angles for various aspect ratios: (a) $a / b=1$, (b) $a / b=2 / 3$ and (c) $a / b=1 / 2$.

Substituting the appropriate expressions of strain energy and potential energy in Eq. (1), and using the assumed approximate displacement fields as given in Eq. (3), the governing equations are obtained in the following form:

$$
[K]\{d\}=\{f\}
$$

where, $[K]=\left[k^{b}\right]+\left[k^{m}\right]$ is the stiffness matrix of dimension $(n w+n u+n v),\left[k^{b}\right]$ is the stiffness matrix due to bending action, $\left[k^{m}\right]$ is the stiffness matrix due to stretching action, $\{d\}$ is the unknown coefficient vector and $\{f\}$ is the load vector. $\left[k^{b}\right]$ and $\left[k^{m}\right]$ are of the form given below:

$$
\left[k^{b}\right]=\left[\begin{array}{lll}
k_{11}^{b} & k_{12}^{b} & k_{13}^{b} \\
k_{21}^{b} & k_{22}^{b} & k_{23}^{b} \\
k_{31}^{b} & k_{32}^{b} & k_{33}^{b}
\end{array}\right] \text { and }\left[k^{m}\right]=\left[\begin{array}{lll}
k_{11}^{m} & k_{12}^{m} & k_{13}^{m} \\
k_{21}^{m} & k_{22}^{m} & k_{23}^{m} \\
k_{31}^{m} & k_{32}^{m} & k_{33}^{m}
\end{array}\right]
$$

The elements of $\left[k^{b}\right]$ and $\left[k^{m}\right]$ are given in the appendix. The load vector $\{f\}$ is of the form $\left[f_{11} f_{12} f_{13}\right]^{T}$, 
where, $\left\{f_{11}\right\}=p \sum_{j=1}^{n w} \int_{0}^{1} \int_{0}^{1} \phi_{j} \operatorname{det} J d \xi d \eta$ and $\left\{f_{12}\right\}=\left\{f_{13}\right\}=0$.

Here, det $J$ is the determinant of the Jacobian matrix of transformation from $x-y$ plane to $\xi-\eta$ plane given by,

$$
J=\left[\begin{array}{ll}
\left(\frac{\partial x}{\partial \xi}\right) & \left(\frac{\partial y}{\partial \xi}\right) \\
\left(\frac{\partial x}{\partial \eta}\right) & \left(\frac{\partial y}{\partial \eta}\right)
\end{array}\right]=\left[\begin{array}{cc}
a & 0 \\
b \sin \theta & b \cos \theta
\end{array}\right] .
$$

The governing equation given by Eq. (4) is non-linear in nature and is solved by direct substitution technique with successive relaxation scheme (Das et al., 2009). The entire computational work is carried out using Compaq Visual Fortran (ver. 6.6).

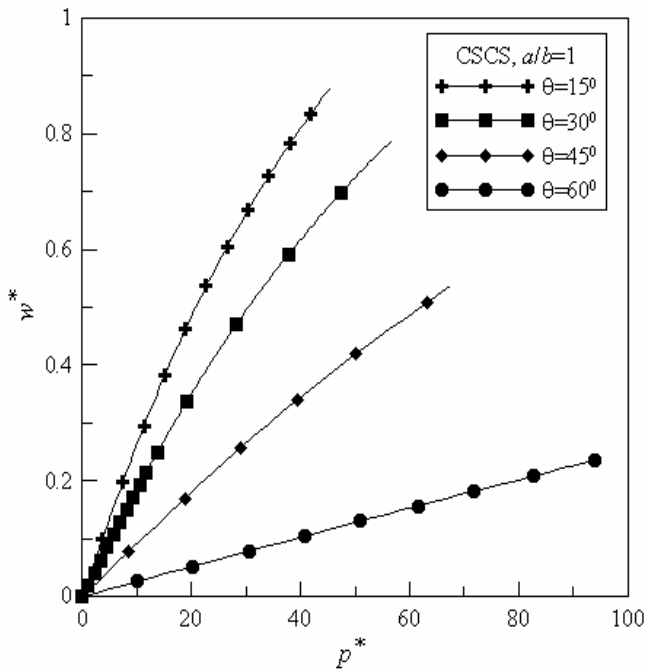

(a) $a / b=1$

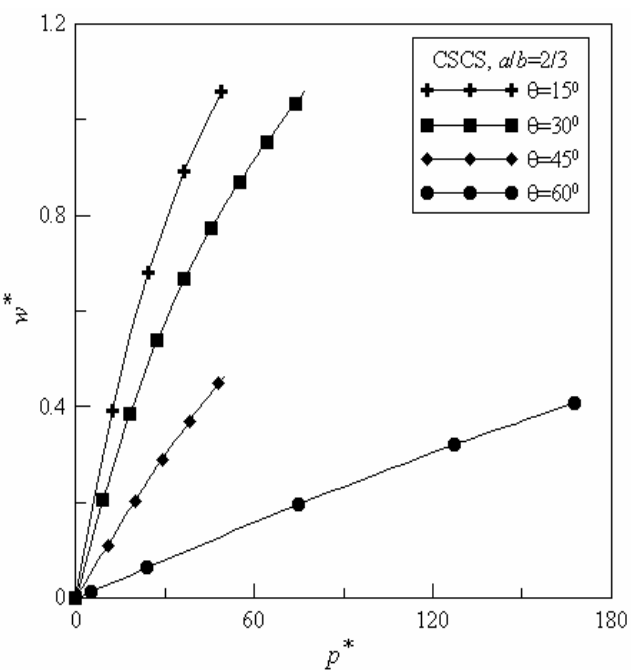

(b) $a / b=2 / 3$

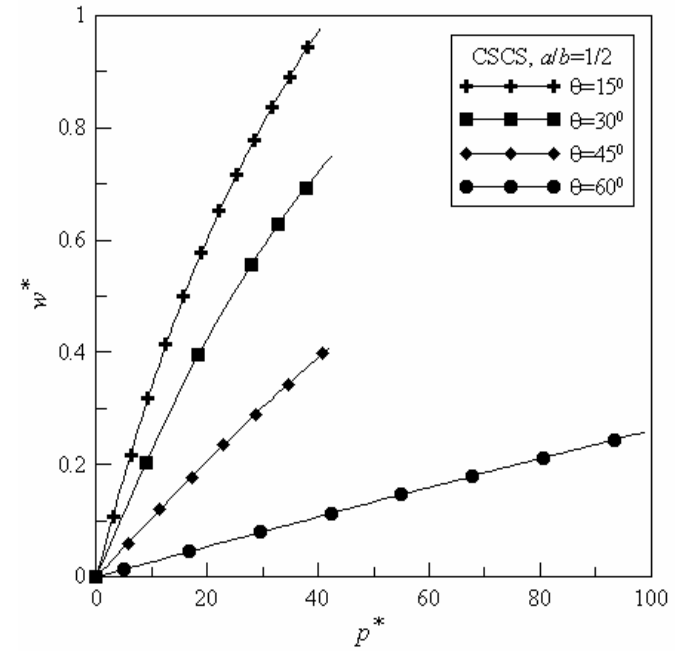

(c) $a / b=1 / 2$

Figure 5. Normalized load-deflection plots of CSCS plate of different skew angles for various aspect ratios: (a) $a / b=1$, (b) $a / b=2 / 3$ and (c) $a / b=1 / 2$. 


\section{Results and Discussion}

Static behaviour of skew plates is presented in the form of load-deflection plots in normalized plane. Results are presented for mixed clamped (C) and simply supported (S) boundary conditions, namely, CCCS, CSCS, CCSS and CSSS. The normalized load parameter is defined as $p^{*}=\left(p a^{4}\right) /(16 D t)$ and the maximum normalized deflection is defined as $w^{*}=w_{\max } / t$. It is to be noted that for symmetric boundary conditions (having opposite symmetric edges), maximum deflection occurs at the plate centre, i.e., the intersection point of the two diagonals, whereas, for asymmetric boundary conditions, the location of maximum deflection needs to be specified separately. The results are generated using the following material and geometric parameter values; $E=210 \mathrm{GPa}, v=$ $1 / 3, a=1.0 \mathrm{~m}$ and $t=0.01 \mathrm{~m}$. The skew angle and the aspect ratio $(a / b)$ of the plate are indicated in the corresponding plots. Convergence and validation of the present approach are shown in the paper by Das et al. (2009) and has not been shown here to maintain brevity.

\subsection{Normalized load-deflection behaviour:}

The normalized load-deflection behaviour of CCCS skew plates has been shown in Figures 4(a)-4(c) for aspect ratio 1, 2/3 and $1 / 2$ respectively. In each of these figures, plots are presented for different values of skew angles and those values are $15^{\circ}, 30^{\circ}, 45^{\circ}$ and $60^{\circ}$. The plots clearly show the non-linear load-deflection behaviour of skew plates, thus showing the effect of geometric nonlinearity. It can also be seen that normalized deflection $\left(w^{*}\right)$ decreases as the skew angle increases for any fixed value of aspect ratio and this behaviour is consistent for all aspect ratios.

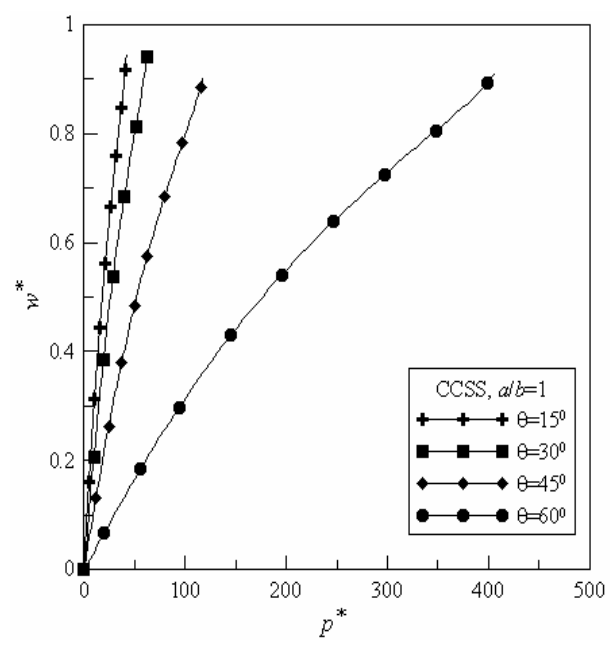

(a) $a / b=1$

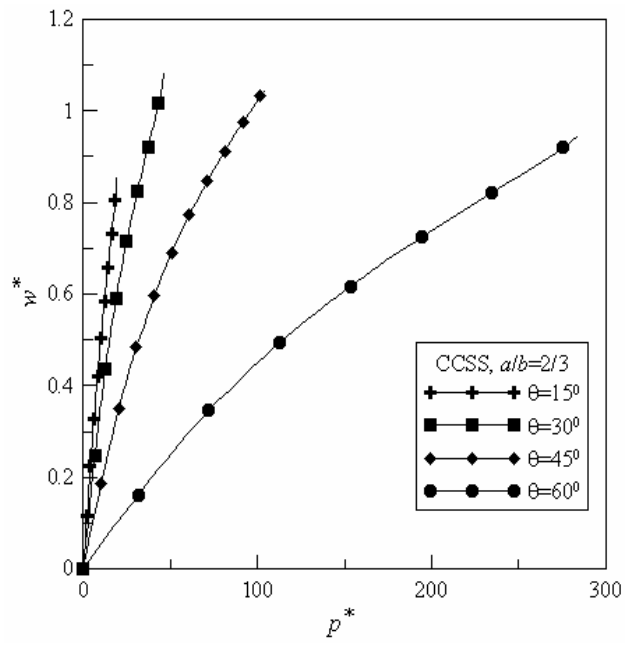

(b) $a / b=2 / 3$

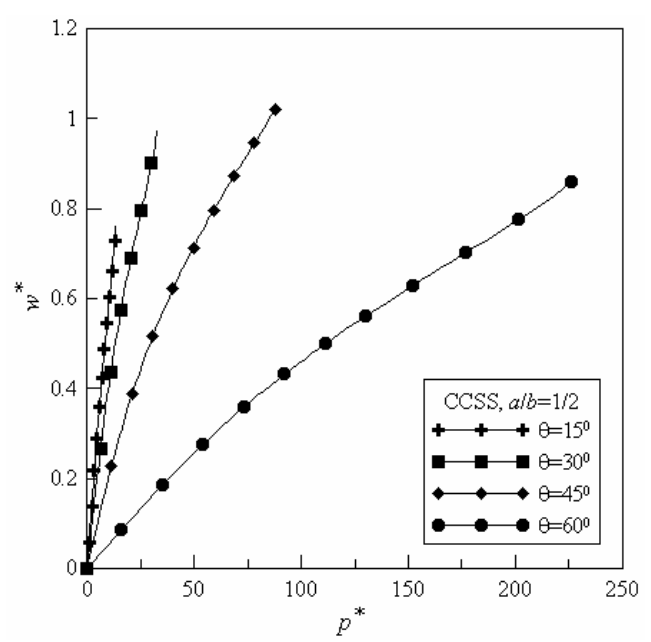

(c) $a / b=1 / 2$

Figure 6. Normalized load-deflection plots of CCSS plate of different skew angles for various aspect ratios: (a) $a / b=1$, (b) $a / b=2 / 3$ and (c) $a / b=1 / 2$. 
The normalized load-deflection behaviour with different skew angles are shown in Figures 5-7 for CSCS, CCSS and CSSS skew plates, respectively, for three different values of various aspect ratios, as considered earlier. The values of the skew angles are also taken as same as that used for CCCS boundary conditions. All these plots exhibit similar trend as observed for CCCS plates. The normalized load deflection behaviour with the variation in aspect ratio have been shown in Figures 8(a-d) for CCCS, CSCS, CCSS and CSSS boundary conditions respectively, for a fixed value of skew angle, $\theta=30^{\circ}$. These plots follow a general consistent trend that for a fixed value of skew angle, the normalized deflection decreases with increases in aspect ratio.

In order to investigate the relative difference in the load-deflection behaviour of skew plates for all the boundary conditions, the normalized load-deflection plots for all the boundary conditions for a rhombic plate having $\theta=30^{\circ}$ are presented in Figure 9 . It is clear from the figure that the magnitude of normalized deflection for any value of normalized load is minimum for CCCS plate and maximum for CSSS plate. The normalized coordinates of the points of maximum deflection in normalized domain corresponding to each boundary condition are also provided in parentheses in the legends. The validation of the load-deflection behaviour for CCCS and CSCS rhombic skew plates having $\theta=30^{\circ}$ with commercial finite element package ANSYS (version 10.0) is also shown in the same figure and the comparison clearly shows the validity of the present method. The results in ANSYS are generated using SHELL63 element with 50 x 50 elements in the two oblique coordinate directions.

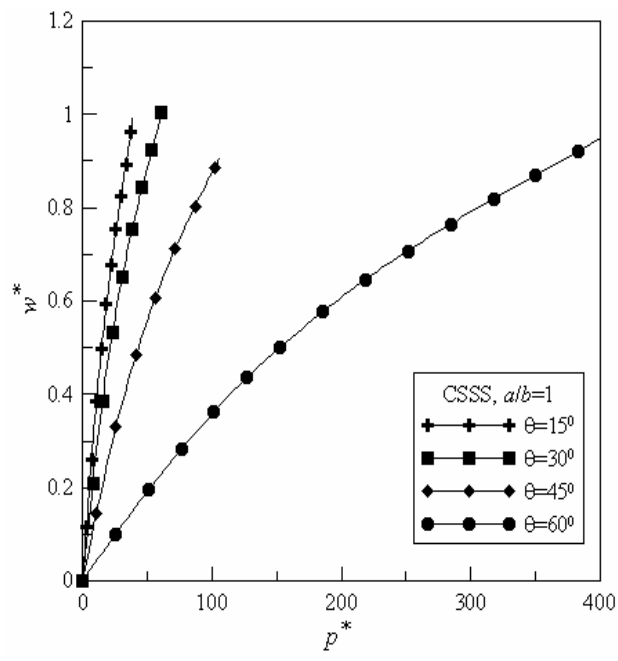

(a) $a / b=1$

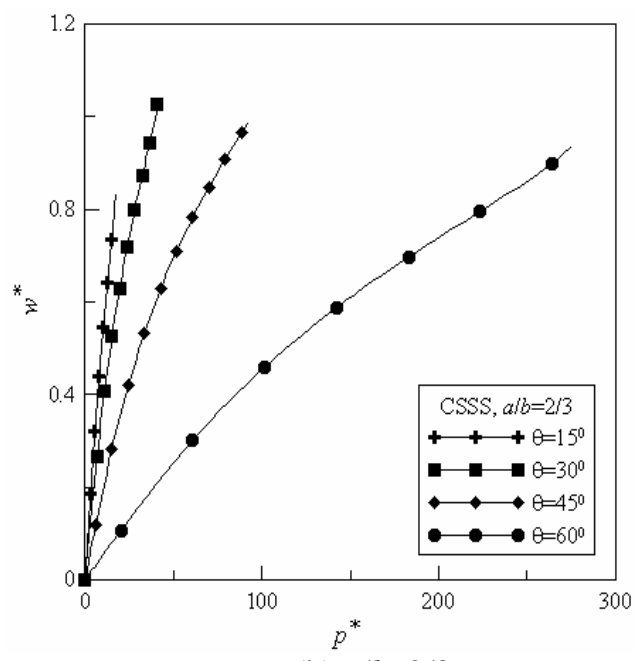

(b) $a / b=2 / 3$

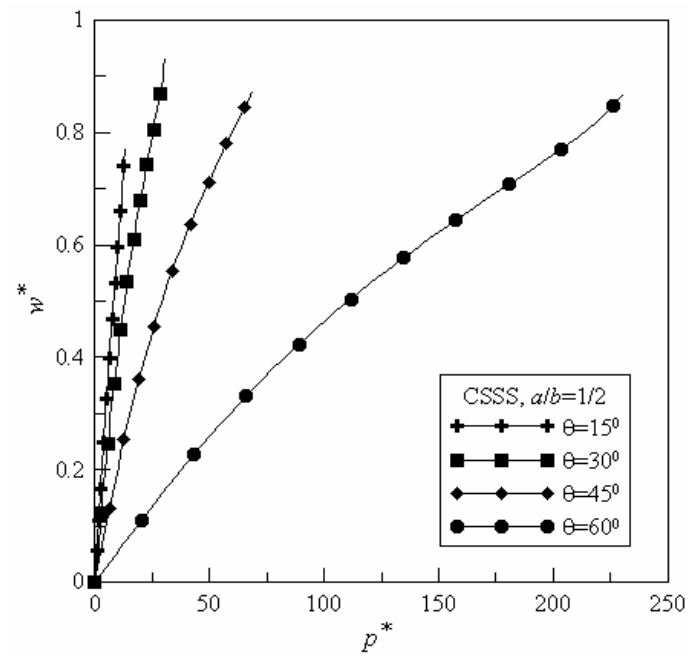

(c) $a / b=1 / 2$

Figure 7. Normalized load-deflection plots of CSSS plate of different skew angles for various aspect ratios: (a) $a / b=1$, (b) $a / b=2 / 3$ and (c) $a / b=1 / 2$. 


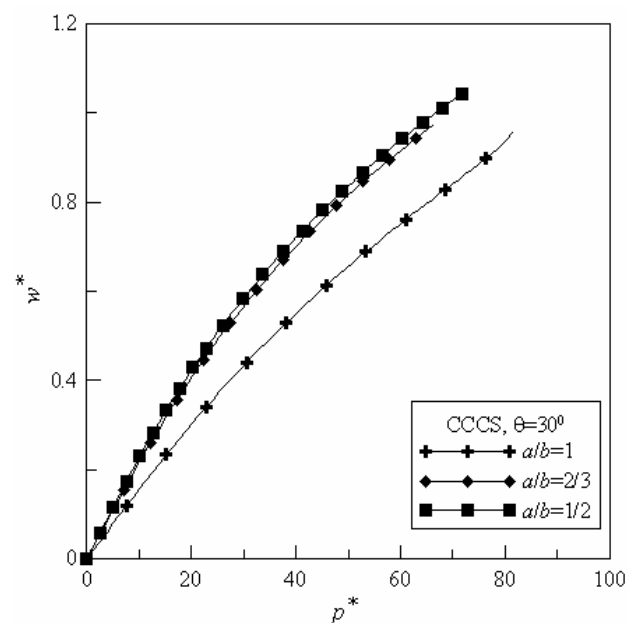

(a) CCCS

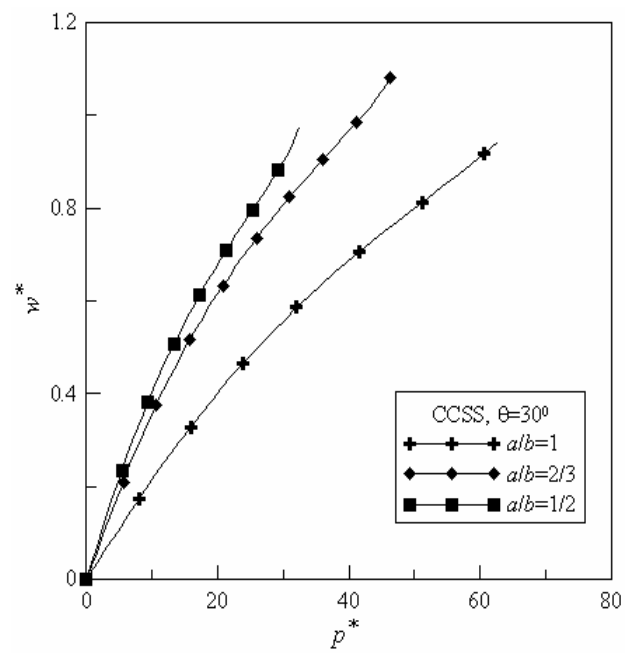

(c) CCSS

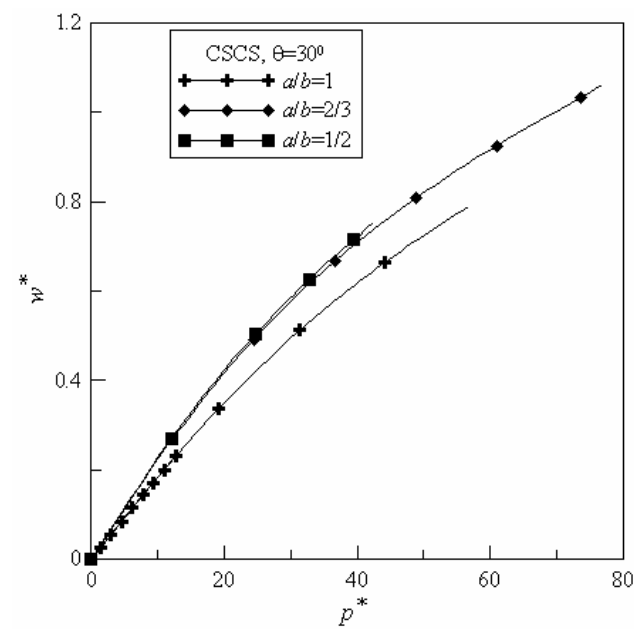

(b) CSCS

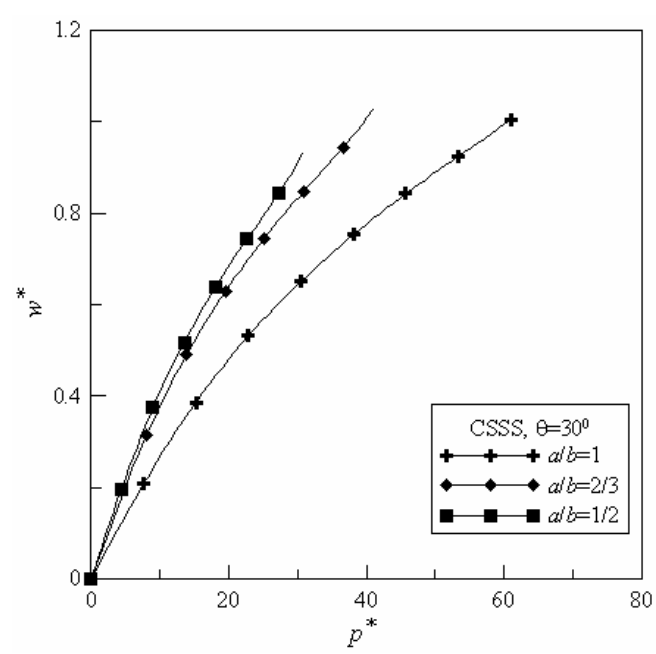

(d) CSSS

Figure 8. Normalized load-deflection plots of $30^{0}$ skew plates for varying aspect ratios having different boundary conditions: (a) CCCS, (b) CSCS, (c) CCSS and (d) CSSS.

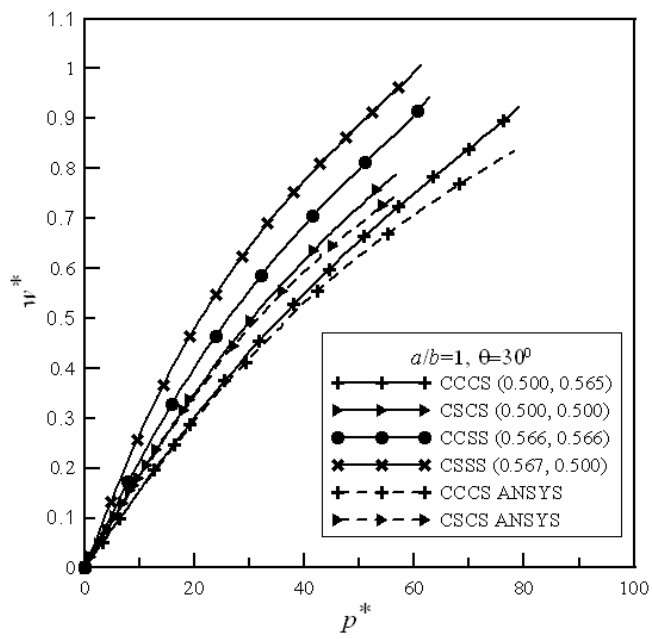

Figure 9. Normalized load-deflection plots of $30^{\circ}$ rhombic plate for different boundary conditions. 


\subsection{Deflected shape:}

In order to visualize the transverse deflection profiles of the skew plates, three dimensional surface and contour plots of deflected shapes of rhombic skew plates for $30^{\circ}$ skew angle have been shown in Figure 10 for all the four boundary conditions. It is to be noted that the deflected shape of the plate is represented by considering only the transverse displacement. Quite obviously, the location of maximum deflection point lies at the plate centre when the boundary conditions are symmetric as can be seen from the contour plots shown in Figure10(b) for CSCS skew plate. For asymmetric boundary conditions, the point of maximum deflection tends to shift away from the clamped boundary which is visible from the contour plots for CCCS, CCSS and CSSS skew plates shown in Figures 10(a), 10(c) and 10(d) respectively. It must be mentioned that the nature of plots shown in Figures 10(a-d) are not dependent on the magnitude of loading and hence they are not to the original scale.

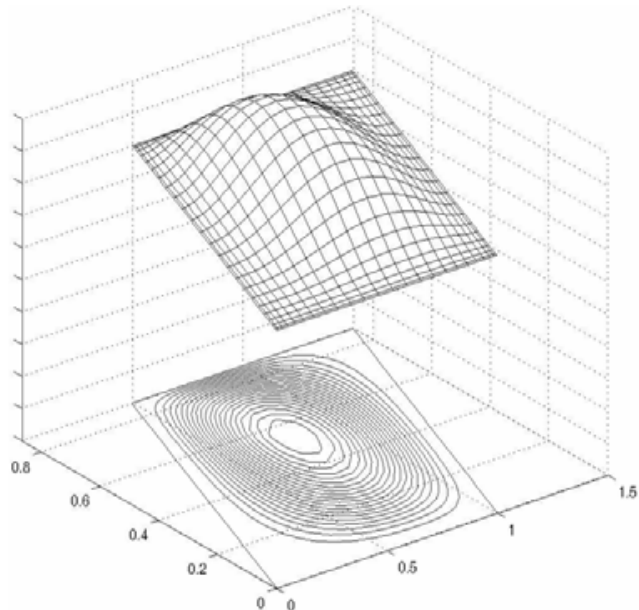

(a) CCCS

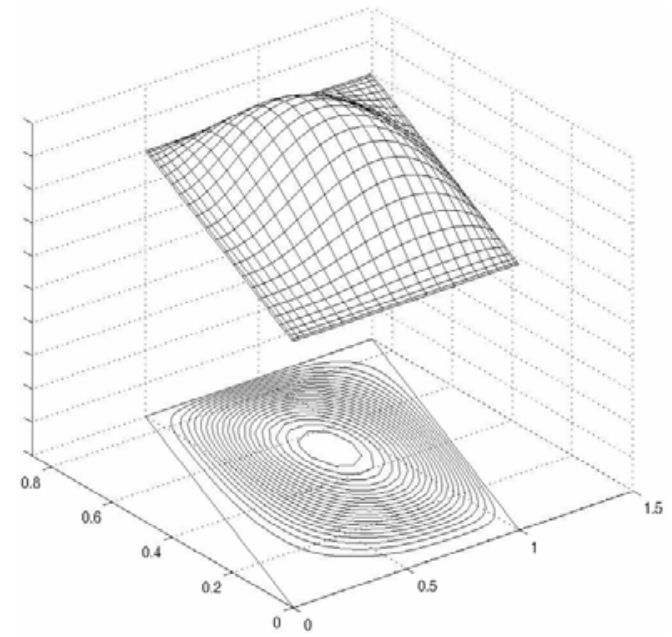

(c) CCSS

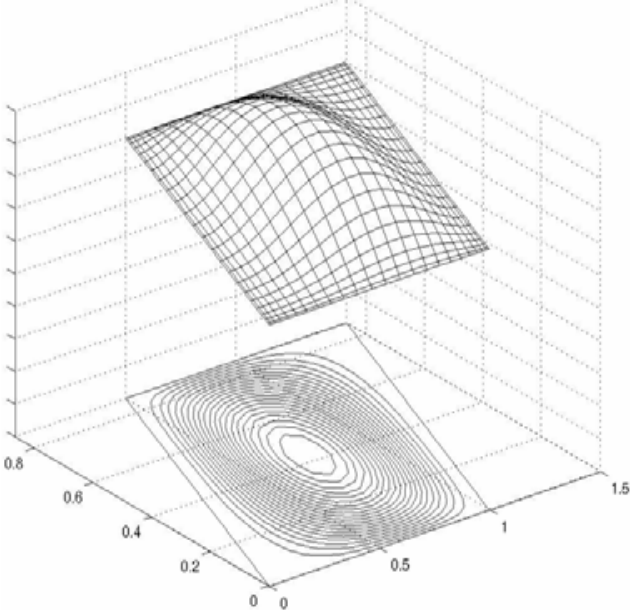

(b) CSCS

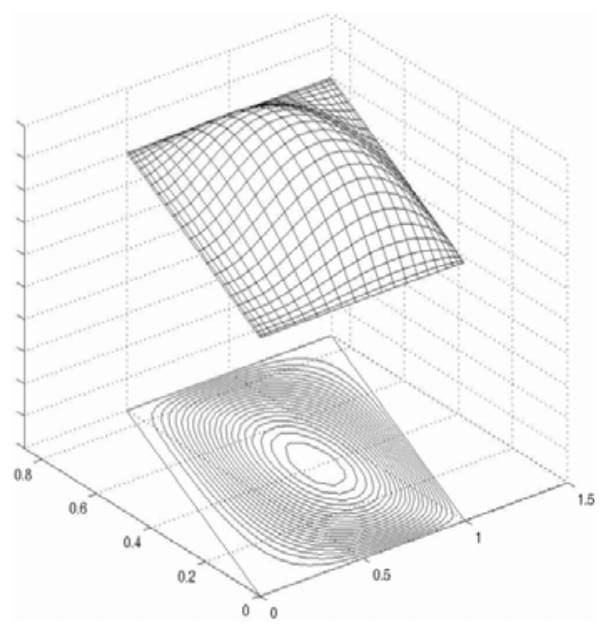

(d) CSSS

Figure 10. Surface and contour plots of deflected shape of rhombic skew plates with $30^{\circ}$ skew angle having boundary conditions: (a) CCCS, (b) CSCS, (c) CCSS and (d) CSSS.

\section{Conclusions}

In this paper, large deflection static analysis of thin isotropic skew plates under uniform transverse pressure has been presented for various mixed flexural boundary conditions. A variational approach has been followed to obtain governing equations and the solution obtained is approximate in nature. Results are presented in the form of normalized load-deflection plots by varying both the skew angles and the aspect ratios, keeping the other fixed. The effect of boundary condition is also shown for a fixed value of aspect ratio and skew angle. Also the deflected shape plots of the plates are shown to visualize the effect of the boundary conditions. The method of analysis which is based on domain mapping technique can be extended to obtain the static and dynamic behavior of irregular shaped structural elements. 


\section{Nomenclature}

a Length of the plate

$b \quad$ Width of plate along skew direction

$\{d\} \quad$ Vector of unknown coefficients

$D \quad$ Flexural rigidity of the plate

E Young's modulus of the plate

$\{f\} \quad$ Load vector

$J \quad$ Jacobian of the coordinate transformation

$[K] \quad$ Stiffness matrix

$\left[k^{b}\right] \quad$ Stiffness matrix due to bending

$\left[k^{m}\right] \quad$ Stiffness matrix due to stretching

$n w \quad$ Number of functions for $w$

$n u \quad$ Number of functions for $u$

$n v \quad$ Number of functions for $v$

$p \quad$ Uniform transverse pressure

$p^{*} \quad$ Non-dimensional load parameter

$t \quad$ Thickness of the plate

$u \quad$ Displacement along $x$ direction

$U \quad$ Strain energy

$U_{b} \quad$ Strain energy due to flexural action

$U_{m} \quad$ Strain energy due to membrane action

$v \quad$ Displacement along $y$ direction

$V \quad$ Work potential

$w \quad$ Transverse displacement

$w^{*} \quad$ Maximum transverse displacement

$v \quad$ Poisson's ratio

$\phi_{i} \quad$ Set of functions defining approximate displacement field $w$

$\alpha_{i} \quad$ Set of functions defining approximate displacement field $u$

$\beta_{i} \quad$ Set of functions defining approximate displacement field $v$

$\theta \quad$ Skew angle

\section{References}

Alwar R.S. and Rao N.R. (1973): Nonlinear analysis of orthotropic skew plates. AIAA Journal, vol.11, pp. 495-498.

Alwar R.S. and Rao N.R. (1974): Large elastic deformations of clamped skewed plates by dynamic relaxation. Computers \& Structures, vol. 4, pp.381-398.

Chia C. (1980): Nonlinear analysis of plates. USA:McGraw-Hill Inc.

Daripa R. and Singha M.K. (2009): Influence of corner stresses on the stability characteristics of composite skew plates. International Journal of Non-Linear Mechanics, vol. 44, No. 2, pp.138-146.

Das D., Sahoo P. and Saha K.N. (2009): A variational analysis for large deflection of skew plates under uniformly distributed load. International Journal Engineering, Science and Technology, vol. 1, pp.16-32.

Duan M. and Mahendran M. (2003): Large deflection analyses of skew plates using hybrid/mixed finite element method. Computers \& Structures, vol.81, pp.1415-1424.

Liew K.M. and Chen X.L. and Reddy J.N. (2004): Mesh-free radial basis function method for buckling analysis of non-uniformly loaded arbitrarily shaped shear deformable plates. Computers Methods in Applied Mechanics and Engineering, vol.193, pp.205224.

Liew K.M. and Han J.B. (1997): Bending analysis of simply supported shear deformable skew plates. Journal of Engineering Mechanics, vol.123, pp.214-221.

Malekzadeh P. and Foiuz A.R. (2007): Large deformation analysis of orthotropic skew plates with nonlinear rotationally restrained edges using DQM. Composite Structures, vol. 80, pp.196-206.

Muhammad T. and Singh A.V. (2004): A p-type solution for the bending of rectangular, circular, elliptic and skew plates. International Journal of Solids and Structures, vol. 41, pp.3977-3997.

Prakash T., Singha M.K. and Ganapathi M. (2008): Thermal postbuckling analysis of FGM skew plates. Engineering Structures, vol. 30, pp.22-32.

Rajamohan C. and Raamachandran J. (1997): Boundary element analysis of skew plates using a new fundamental solution. Mechanics Research Communications, vol.24, pp.407-414. 
Singh A.V. and Elaghabash Y. (2003): On finite displacement analysis of quadrangular plates. International Journal of NonLinear Mechanics, vol.38, pp.1149-1162.

Xiang Y., Wang C.M. and Kitipornchai S. (1995): Buckling of skew Mindlin plates subjected to in-plane shear loadings. International Journal of Mechanical Sciences, vol.37, pp.1089-1101.

\section{Appendix}

$$
\begin{aligned}
& {\left[k_{11}^{b}\right]=D \sum_{j=1}^{n w} \sum_{i=1}^{n w} \int_{0}^{1} \int_{0}^{1}\left[\left\{\left(\frac{\partial^{2} \phi_{i}}{\partial x^{2}}\right)\left(\frac{\partial^{2} \phi_{j}}{\partial x^{2}}\right)+\left(\frac{\partial^{2} \phi_{i}}{\partial y^{2}}\right)\left(\frac{\partial^{2} \phi_{j}}{\partial y^{2}}\right)+\left(\frac{\partial^{2} \phi_{i}}{\partial y^{2}}\right)\left(\frac{\partial^{2} \phi_{j}}{\partial x^{2}}\right)\right.\right.} \\
& \left.+\left(\frac{\partial^{2} \phi_{i}}{\partial x^{2}}\right)\left(\frac{\partial^{2} \phi_{j}}{\partial y^{2}}\right)\right\}-(1-v)\left\{\left(\frac{\partial^{2} \phi_{i}}{\partial y^{2}}\right)\left(\frac{\partial^{2} \phi_{j}}{\partial x^{2}}\right)+\left(\frac{\partial^{2} \phi_{i}}{\partial x^{2}}\right)\left(\frac{\partial^{2} \phi_{j}}{\partial y^{2}}\right)\right. \\
& \left.\left.-2\left(\frac{\partial^{2} \phi_{i}}{\partial x \partial y}\right)\left(\frac{\partial^{2} \phi_{j}}{\partial x \partial y}\right)\right\}\right] \operatorname{det} J d \xi d \eta \\
& {\left[k_{12}^{b}\right]=\left[k_{13}^{b}\right]=\left[k_{21}^{b}\right]=\left[k_{22}^{b}\right]=\left[k_{23}^{b}\right]=\left[k_{31}^{b}\right]=\left[k_{32}^{b}\right]=\left[k_{33}^{b}\right]=0,} \\
& {\left[k_{11}^{m}\right]=\frac{E t}{2\left(1-v^{2}\right)} \sum_{j=1}^{n w} \sum_{i=1}^{n w} \int_{0}^{1} \int_{0}^{1}\left[\left(\sum_{i=1}^{n w} d_{i} \frac{\partial \phi_{i}}{\partial x}\right)^{2} \frac{\partial \phi_{i}}{\partial x} \frac{\partial \phi_{j}}{\partial x}+\left(\sum_{i=1}^{n w} d_{i} \frac{\partial \phi_{i}}{\partial y}\right)^{2} \frac{\partial \phi_{i}}{\partial y} \frac{\partial \phi_{j}}{\partial y}\right.} \\
& +\left(\sum_{i=1}^{n w} d_{i} \frac{\partial \phi_{i}}{\partial x}\right)\left(\sum_{i=1}^{n w} d_{i} \frac{\partial \phi_{i}}{\partial y}\right)\left\{\frac{\partial \phi_{i}}{\partial x} \frac{\partial \phi_{j}}{\partial y}+\frac{\partial \phi_{i}}{\partial y} \frac{\partial \phi_{j}}{\partial x}\right\}+(1-v)\left(\sum_{i=n w+1}^{n w+n u} d_{i} \frac{\partial \alpha_{i-n w}}{\partial y}\right) \frac{\partial \phi_{i}}{\partial x} \frac{\partial \phi_{j}}{\partial y} \\
& \left.+(1-v)\left(\sum_{i=n w+n u+1}^{n w+n u+n v} d_{i} \frac{\partial \beta_{i-n w-n u}}{\partial x}\right) \frac{\partial \phi_{i}}{\partial x} \frac{\partial \phi_{j}}{\partial y}\right] \operatorname{det} J d \xi d \eta, \\
& {\left[k_{12}^{m}\right]=\frac{E t}{2\left(1-v^{2}\right)} \sum_{j=1}^{n w} \sum_{i=n w+1}^{n w+n u} \int_{0}^{1} \int_{0}^{1}\left[2\left(\sum_{i=1}^{n w} d_{i} \frac{\partial \phi_{i}}{\partial x}\right) \frac{\partial \alpha_{i-n w}}{\partial x} \frac{\partial \phi_{j}}{\partial x}+2 v\left(\sum_{i=1}^{n w} d_{i} \frac{\partial \phi_{i}}{\partial y}\right) \frac{\partial \alpha_{i-n w}}{\partial x} \frac{\partial \phi_{j}}{\partial y}\right.} \\
& \left.+(1-v)\left(\sum_{i=1}^{n w} d_{i} \frac{\partial \phi_{i}}{\partial y}\right) \frac{\partial \alpha_{i-n w}}{\partial y} \frac{\partial \phi_{j}}{\partial x}\right] \operatorname{det} J d \xi d \eta, \\
& {\left[k_{13}^{m}\right]=\frac{E t}{2\left(1-v^{2}\right)} \sum_{j=1}^{n w} \sum_{i=n w+n u+1}^{n w+n u+n v} \int_{0}^{1} \int_{0}^{1}\left[2\left(\sum_{i=1}^{n w} d_{i} \frac{\partial \phi_{i}}{\partial y}\right) \frac{\partial \beta_{i-n w-n u}}{\partial y} \frac{\partial \phi_{j}}{\partial y}+2 v\left(\sum_{i=1}^{n w} d_{i} \frac{\partial \phi_{i}}{\partial x}\right) \frac{\partial \beta_{i-n w-n u}}{\partial y} \frac{\partial \phi_{j}}{\partial x}\right.} \\
& \left.+(1-v)\left(\sum_{i=1}^{n w} d_{i} \frac{\partial \phi_{i}}{\partial y}\right) \frac{\partial \beta_{i-n w-n u}}{\partial x} \frac{\partial \phi_{j}}{\partial x}\right] \operatorname{det} J d \xi d \eta, \\
& {\left[k_{21}^{m}\right]=\frac{E t}{2\left(1-v^{2}\right)} \sum_{j=n w+1}^{n w+n u} \sum_{i=1}^{n w} \int_{0}^{1} \int_{0}^{1}\left[2\left(\sum_{i=1}^{n w} d_{i} \frac{\partial \phi_{i}}{\partial x}\right) \frac{\partial \phi_{i}}{\partial x} \frac{\partial \alpha_{j-n w}}{\partial x}+v\left(\sum_{i=1}^{n w} d_{i} \frac{\partial \phi_{i}}{\partial y}\right) \frac{\partial \phi_{i}}{\partial y} \frac{\partial \alpha_{j-n w}}{\partial x}\right.} \\
& \left.+(1-v)\left(\sum_{i=1}^{n w} d_{i} \frac{\partial \phi_{i}}{\partial x}\right) \frac{\partial \phi_{i}}{\partial y} \frac{\partial \alpha_{j-n w}}{\partial y}\right] \operatorname{det} J d \xi d \eta, \\
& {\left[k_{22}^{m}\right]=\frac{E t}{2\left(1-v^{2}\right)} \sum_{j=n w+1}^{n w+n u} \sum_{i=n w+1}^{n w+n u} \int_{0}^{1} \int_{0}^{1}\left[2 \frac{\partial \alpha_{i-n w}}{\partial x} \frac{\partial \alpha_{j-n w}}{\partial x}+(1-v) \frac{\partial \alpha_{i-n w}}{\partial y} \frac{\partial \alpha_{j-n w}}{\partial y}\right] \operatorname{det} J d \xi d \eta} \\
& {\left[k_{23}^{m}\right]=\frac{E t}{2\left(1-v^{2}\right)} \sum_{j=n w+1}^{n w+n u} \sum_{i=n w+n u+1}^{n w+n u+n v} \int_{0}^{1} \int_{0}^{1}\left[2 v \frac{\partial \beta_{i-n w-n u}}{\partial y} \frac{\partial \alpha_{j-n w}}{\partial x}\right.} \\
& \left.+(1-v) \frac{\partial \beta_{i-n w-n u}}{\partial x} \frac{\partial \alpha_{j-n w}}{\partial y}\right] \operatorname{det} J d \xi d \eta,
\end{aligned}
$$




$$
\begin{aligned}
& {\left[k_{31}^{m}\right]=} \frac{E t}{2\left(1-v^{2}\right)} \sum_{j=n w+n u+1}^{n w+n u+n v} \sum_{i=1}^{n w} \int_{0}^{1} \int_{0}^{1}\left[2\left(\sum_{i=1}^{n w} d_{i} \frac{\partial \phi_{i}}{\partial y}\right) \frac{\partial \phi_{i}}{\partial y} \frac{\partial \beta_{j-n w-n u}}{\partial y}+v\left(\sum_{i=1}^{n w} d_{i} \frac{\partial \phi_{i}}{\partial x}\right) \frac{\partial \phi_{i}}{\partial x} \frac{\partial \beta_{j-n w-n u}}{\partial y}\right. \\
&\left.+(1-v)\left(\sum_{i=1}^{n w} d_{i} \frac{\partial \phi_{i}}{\partial x}\right) \frac{\partial \phi_{i}}{\partial y} \frac{\partial \beta_{j-n w-n u}}{\partial x}\right] \operatorname{det} J d \xi d \eta, \\
& {\left[k_{32}^{m}\right]=} \frac{E t}{2\left(1-v^{2}\right)} \sum_{j=n w+n u+1}^{n w+n u+n v} \sum_{i=n w+1}^{n w+n u} \int_{0}^{1} \int_{0}^{1}\left[2 v \frac{\partial \alpha_{i-n w}}{\partial x} \frac{\partial \beta_{j-n w-n u}}{\partial y}\right. \\
&\left.+(1-v) \frac{\partial \alpha_{i-n w}}{\partial y} \frac{\partial \beta_{j-n w-n u}}{\partial x}\right] \operatorname{det} J d \xi d \eta \\
& \text { and }\left[k_{33}^{m}\right]=\frac{E t}{2\left(1-v^{2}\right)} \sum_{j=n w+n u+1 i=n w+n u+1}^{n w+n u+n v} n \int_{0}^{n w+n u+n v} \int_{0}^{1}\left[2 \frac{\partial \beta_{j-n w-n u}}{\partial y} \frac{\partial \beta_{j-n w-n u}}{\partial y}\right. \\
&\left.\quad+(1-v) \frac{\partial \beta_{j-n w-n u}}{\partial x} \frac{\partial \beta_{j-n w-n u}}{\partial x}\right] \operatorname{det} J d \xi d \eta .
\end{aligned}
$$

Here, $D=\frac{E t^{3}}{12\left(1-v^{2}\right)}$ is the flexural rigidity of the plate.

\section{Biographical notes}

Debabrata Das graduated from the Department of Mechanical Engineering, R. E. C., Durgapur, India and got his Master in Mechanical Engineering degree from Jadavpur University, Kolkata, India. He received Ph. D. degree in Mechanical Engineering from Jadavpur University, Kolkata, India. He has served the national Defence Research and Development Organization for five years as a scientist. Thereafter he started pursuing research in the area of structural mechanics. He has 15 publications in international journals and conference proceedings. At present he serves as Lecturer in Jadavpur University, Kolkata, India.

Prasanta Sahoo is Professor at the Department of Mechanical Engineering, Jadavpur University, Kolkata, India. He received Bachelor of Mechanical Engineering from Jadavpur University and MTech and PhD in Mechanical Engineering from Indian Institute of Technology, Kharagpur, India. His research area is tribology and structural mechanics. He is the supervisor of $7 \mathrm{PhD}$ theses and 16 Master's theses. He has authored a textbook on Engineering Tribology (Prentice Hall of India, 2005) and book-chapters in Tribology Research Advances (Nova Science, 2009) and Tribology for Engineers - a practical guide (Woodhead Publishing, 2010). He has co-authored more than 150 technical papers in international journals and conference proceedings. He is on the editorial board of International Journal of Engineering, Science and Technology (Multicraft), Journal of Tribology Research (Serials), Journal of Tribology and Surface Engineering (Nova Science), International Journal of Manufacturing, Materials and Mechanical Engineering (IGI Global), Materials and Design (Elsevier).

Kashinath Saha graduated from the Department of Mechanical Engineering, Jadavpur University and then served Indian industry for five years as design engineer. Joined academia as lecturer in the Department of Mechanical Engineering, Jadavpur University in 1987 and pursuing teaching and research in the area of Machine Elements Design and Computer aided drafting, Stress, deformation analysis and design of Structural Elements and Structural Stability Analysis. He has also carried out several experimental studies in the field of structural dynamics and supervised a number of post graduate thesis and research projects and has over 50 publications in international journals and conference proceedings. At present he serves as Associate Professor in the Department of Mechanical Engineering, Jadavpur University.

Received December 2009

Accepted March 2010

Final acceptance in revised form May 2010 